\title{
Do appointment reminders work?
}

\author{
The use of postal reminders to reduce non-attendance at an orthodontic clinic: A randomised controlled trial \\ S. Can, T. Macfarlane and K. D. O'Brien Br Dent J 2003; 195: 199-201
}

\section{Objectives}

To evaluate the effect of issuing a patient reminder plus a confirmation slip on the attendance of orthodontic new patients.

\section{Setting}

Department of Orthodontics, University Dental Hospital of Manchester.

Design

A randomised controlled trial.

\section{Method}

New patients were randomly allocated to:

i) receive a reminder letter and return a confirmation slip or

ii) not receive a reminder.

\section{Outcome measures}

Patient attendance at the clinic.

Results

A total of 232 patients were entered into the study between $18 / 06 / 2001$ and $29 / 08 / 2001$. These were randomly allocated to $115(49.8 \%)$ in the reminder group and $116(50.2 \%)$ in the no reminder group. If the patient received a reminder and returned the confirmation they were less likely to fail the appointment than if they did not receive a reminder (OR $0.4,95 \%$ CI 0.2 to 0.96 ) There was an effect of social deprivation, if the patients lived in an area of high social deprivation they were 2.7 (95\% CI 1.1 to 6.5) times more likely to fail to attend an appointment than people who were more affluent.

\section{Conclusions}

The use of postal reminders for orthodontic consultation appointments appears to result in a useful increase of appointments that are kept or cancelled in advance.

\section{IN BRIEF}

- The use of postal reminders for orthodontic consultation appointments appears to result in a useful increase of appointments that are kept or cancelled in advance

- If a patient receives a reminder and does not return the confirmation slip, there is a 33\% chance that they will not attend.

- The use of a reminder does not counteract the effect of social deprivation on non-attendance at the clinic.

\section{COMMENT}

The hospital service has always been the most expensive part of the NHS and it is therefore only natural that attention should be focused on the most effective use of this resource. When all hospital outpatient departments are considered, in 2000/2001 in England over 1.5 million patients failed to attend their first appointment while almost 0.5 million failed to attend subsequent appointments. ${ }^{1}$ It has been estimated that each failed appointment costs the NHS a minimum of $£ 50$. This means that missed hospital outpatient appointments cost the NHS approximately $€ 280 \mathrm{~m}$ per year. They also add increased pressure to waiting list management and mean that patients do not receive treatment.

This study explores one way of reducing non-attendance rates at a dental hospital orthodontic department. The authors completed a randomised controlled trial where the test group were sent a reminder letter two weeks before their appointments were due. The reminder letter was sent with a stamped addressed postcard, which the patients returned to confirm their appointment. The authors report that those patients who returned the confirmation postcard were almost twice as likely to keep their appointment than the patients who did not receive a reminder. Female patients and those from socially deprived areas were also more likely to fail to keep their appointment.

This paper provides an important insight into the problem of non-attendance at hospital orthodontic departments and has a useful message for all clinicians. Issues not explored in this paper include a comparison of the length of time the patients had to wait for their appointment and non-attendance rates. Research has shown that non-attendance rates increase with the length of time a patient has been waiting to be seen. There is also evidence of the need for a greater understanding of the patient's perspective. It may not always be evident to those initiating outpatient appointments that some patients experience considerable difficulties in making arrangements to travel to a dental hospital clinic. Patients with their own cars face difficulties if the hospital has inadequate parking space, those who depend on public transport can be affected by the cost or inconvenience of the service.

Donald Burden, Department of Orthodontics

Queen's University, Belfast

doi:10.1038/sj.bdj.4810440

1. www.doh.gov.uk/hospitalactivity/statistics/2000-01 